\title{
Vitamin D Deficiency in Axial Spondyloarthritis is Associated With Higher Disease Activity
}

\author{
Sizheng ZHAO, ${ }^{1}$ Daniel THONG,${ }^{2}$ Stephen DUFFIELD, ${ }^{1}$ Nicola GOODSON ${ }^{1}$ \\ ${ }^{1}$ Department of Musculoskeletal Biology I, University of Liverpool Institute of Ageing and Chronic Disease, Liverpool, United Kingdom \\ ${ }^{2}$ Department of Medical Education, University of Liverpool, Liverpool, United Kingdom
}

\begin{abstract}
Objectives: This study aims to assess whether vitamin $D$ deficiency is associated with increased disease activity and functional impairment in axial spondyloarthritis (axSpA), with control for its seasonal variation.

Patients and methods: Serum 25-hydroxyvitamin D [25(OH)D] levels were measured in 235 consecutive axSpA patients (176 males, 59 females; mean age 46.3 years; range 18 to 85 years) attending a specialist spondyloarthritis service in the United Kingdom. Disease activity and functional status were assessed using Bath Ankylosing Spondylitis indices, C-reactive protein, and erythrocyte sedimentation rates. Vitamin D deficiency was defined as $25(\mathrm{OH}) \mathrm{D}<30 \mathrm{nmol} / \mathrm{L}$. Associations between vitamin D deficiency and: (i) disease activity (Bath Ankylosing Spondylitis Disease Activity Index), (ii) spinal pain, (iii) functional impairment (Bath Ankylosing Spondylitis Functional Index), and (iv) inflammatory markers were explored using multivariable logistic regression models (adjusted for age, sex, vitamin D supplementation, and seasonal variation).

Results: Median symptom duration was 17 years (inter-quartile range 8.5 to 28.6 years). Median 25(OH)D was $54.5 \mathrm{nmol} / \mathrm{L}$ (inter-quartile range 34 to $77 \mathrm{nmol} / \mathrm{L}$ ) and 52 patients (22\%) were deficient for vitamin D. Increasing Bath Ankylosing Spondylitis Disease Activity Index (adjusted odds ratio 1.23; 95\% confidence interval 1.06-1.41), spinal pain visual analog scale (adjusted odds ratio 1.21; 95\% confidence interval 1.07-1.38), and C-reactive protein (adjusted odds ratio 1.02; $95 \%$ confidence interval 1.01-1.04) were each significantly associated with 25(OH)D deficiency.

Conclusion: This cross-sectional study demonstrated associations between vitamin D deficiency and both higher disease activity and functional impairment in axSpA. Whilst this may reflect reduced ultra-violet exposure in functionally impaired patients, it supports the hypothesis that vitamin $D$ may have an immunomodulatory role. Interventional studies are needed to evaluate a potential causal relationship, as optimizing vitamin $D$ may be a cost-effective adjunctive intervention to modify disease activity in axSpA.

Keywords: Ankylosing spondylitis; axial spondyloarthritis; serum 25-hydroxyvitamin D; vitamin D deficiency.
\end{abstract}

In axial spondyloarthritis (axSpA), chronic inflammation is associated with accelerated loss of bone mineral density and new bone formation. ${ }^{1}$ Although there have been developments in understanding the role of inflammation on bone loss, subsequent abnormal bone remodeling in axSpA remains poorly understood.

Vitamin $D$ is important for calcium homeostasis and bone health, but also has immunomodulatory effects. Many immune cells can convert 25-hydroxyvitamin D [25(OH)D] to its active metabolite, 1,25-dihydroxyvitamin $\mathrm{D}$
$[1,25(\mathrm{OH}) 2 \mathrm{D}]$, which has been shown to inhibit T-helper 17 (Th17) and Th1 cell activity, and promote regulatory T-cells and Th2 cells. ${ }^{2}$

Axial spondyloarthritis is associated with reduced serum 25(OH)D levels when compared with healthy controls. ${ }^{3}$ However, it is not clear from previous studies whether 25(OH)D levels are associated with increased disease activity and functional impairment. This is in part due to inherent difficulties in studying serum levels, as ultraviolet B (UVB) generation of 25(OH)D follows seasonal variation. ${ }^{4}$ Previous cross-sectional 
studies, exploring disease association with vitamin $\mathrm{D}$ levels in axSpA, were not adjusted for season of testing. ${ }^{3}$

Given the potential for vitamin $\mathrm{D}$ to influence both bone metabolism and the immune system, it is hypothesized that vitamin D may influence axSpA disease activity. Therefore, in this study, we aimed to assess whether vitamin D deficiency is associated with increased disease activity and functional impairment in axSpA, with control for its seasonal variation.

\section{PATIENTS AND METHODS}

A total of 235 patients (176 males, 59 females; mean age 46.3 years; range 18 to 85 years) attending a spondyloarthritis service in Aintree University Hospital, UK between November 2011 and November 2015, with a diagnosis of axSpA (assessed using modified New York criteria for ankylosing spondylitis or Assessment of SpondyloArthritis International Society Criteria for axSpA), 5,6 were consecutively recruited for the study. The clinical data were collected during routine out-patient assessments. Patient characteristics (age, sex, smoking status -recorded as ever or never smoking- and body mass index) were recorded at the time of assessment. In addition, disease variables were recorded including symptom duration, duration since diagnosis, human leukocyte antigen B27 (HLA-B27) status if available, and extra-articular disease features. Current use of non-steroidal anti-inflammatory drugs, tumor necrosis factor inhibitors, and any vitamin D supplementation were also recorded. Disease activity and functional status were assessed using the Bath Ankylosing Spondylitis Disease Activity Index (BASDAI), spinal pain visual analog scale (spVAS), Bath Ankylosing Spondylitis Functional Index (BASFI), and the modified Health Assessment Questionnaire (HAQ) disability index. ${ }^{7,8}$ Blood samples were taken in the morning on the day of assessment for analysis of erythrocyte sedimentation rate (ESR), C-reactive protein (CRP), and 25(OH)D, which was measured by tandem mass spectrometry. The date of assessment was recorded.

Vitamin D deficiency was defined as $25(\mathrm{OH}) \mathrm{D}$ $<30 \mathrm{nmol} / \mathrm{L}$. ${ }^{9}$ The study protocol was approved by Aintree University Hospital and the LondonBrent Research Ethics Committee (15/LO/1519). A written informed consent was obtained from each patient. The study was conducted in accordance with the principles of the Declaration of Helsinki.

\section{Statistical analysis}

Statistical analyses were performed using Stata $12^{\circledR}$ (Stata Corp., Texas, USA). Target sample size was calculated at 250 based on an estimate of vitamin D deficiency prevalence of $20 \%$ and using BASDAI as the primary outcome variable with four additional covariates. Vitamin D deficient patients were compared with non-deficient patients using Student's t-test for Gaussian, Mann-Whitney U for non-Gaussian, and chi-squared test for categorical variables. Univariate logistic regression was used to explore the association between vitamin $\mathrm{D}$ deficiency and (i) BASDAI, (ii) spVAS, (iii) BASFI, and (iv) inflammatory markers (CRP and ESR). Variables with significant association were then entered into multivariable models adjusting for age, sex, vitamin $\mathrm{D}$ supplementation, and seasonal variation. Secondary analyses were also performed using increasing tertiles of each marker of disease severity. Given that UVB exposure (and vitamin D production) is likely to be reduced in those with impaired function, sensitivity analyses were performed to investigate whether stratification by functional impairment influenced association between 25(OH)D deficiency and disease activity. The median BASFI in the cohort was used to stratify the sample into low and high functional impairment.

To adjust for seasonal variation in vitamin $D$ deficiency, a sine function was generated: $F(t)=\alpha^{*} \cos \left(2 \pi^{*} t / 365-\theta\right)$, where $t=$ day of the year, $\alpha=$ amplitude, and horizontal shift $\theta=2 \pi^{*} t_{\max } / 365$. Values for $\alpha$ and $t_{\max }$ were calculated from coefficients of a sine term, $\beta_{\sin }{ }^{*} \sin (2 \pi * t / 365)$, and cosine term, $\beta_{\cos }{ }^{*} \cos (2 \pi * t / 365)$, included in the logit regression model of vitamin $\mathrm{D}$ deficiency and BASDAI, where $\alpha=\sqrt{ }\left(\beta_{\sin ^{2}}{ }^{2}+\beta_{\cos ^{2}}{ }^{2}\right)=1.14$ and $\quad t_{\max }=\arctan \left(\beta_{\sin } / \beta_{\cos }\right) * 365 / 2 \pi=40.3$. Logit regression with vitamin $\mathrm{D}$ deficiency as dependent variable and this sine function as independent variable give a coefficient of $\beta=1.00$ (95\% confidence interval [CI] 0.55-1.45). Detailed methodology was described by Stolwijk et al. ${ }^{10}$ 


\section{RESULTS}

The cohort was predominantly male (75\%) and Caucasian (97\%) with median symptom duration of 17 years (inter-quartile range 8.5 to 28.6 years) and median duration since diagnosis of 4.3 years (inter-quartile range 0.8 to 14.9 years). Non-steroidal anti-inflammatory drugs were used by 163 patients (69\%) and $74(31 \%)$ were treated with tumor necrosis factor inhibitors. Patient demographics and disease characteristics are shown in Table 1.

The median 25(OH)D level was $54.5 \mathrm{nmol} / \mathrm{L}$ (inter-quartile range $34-77 \mathrm{nmol} / \mathrm{L}$ ). Deficiency for vitamin D was found in 47 (20\%) patients. Vitamin D supplementation was used by 60 patients (26\%). No associations were observed between vitamin $\mathrm{D}$ supplementation and vitamin D deficiency. Patients deficient for vitamin D had both shorter median symptom duration and duration since diagnosis. Deficient patients were more commonly ever-smokers (55\% vs. $41 \%)$ although this difference was not statistically significant. Median BASDAI (7.2 vs. 5.5), spVAS ( 8 vs. 5$)$, ESR (10 vs. $8 \mathrm{~mm} / \mathrm{hr}$ ), and CRP (5 vs. $3 \mathrm{mg} / \mathrm{L}$ ) were all significantly higher in patients deficient for vitamin $\mathrm{D}$ (Table 1). No differences were observed for BASFI or HAQ.

Peripheral joint involvement was reported in $26 \%$ of patients and $9 \%$ had associated inflammatory bowel disease. There was a trend towards greater proportion with psoriasis in the deficient group compared to non-deficient ( $27 \%$ vs. $12 \%$ ).

Univariate logistic regression revealed that vitamin D deficiency was associated with BASDAI, spVAS, ESR, and CRP, but not BASFI (Table 2). Associations remained significant in multivariate models for BASDAI (adjusted odds ratio $\left[\mathrm{OR}_{\mathrm{adj}}\right]$ 1.23; 95\% CI, 1.06-1.41), spVAS (OR adj 1.21; 95\% CI, 1.07-1.38), and CRP (OR adj 1.02; 95\% CI, 1.01-1.04) adjusted for age, sex, vitamin D supplementation, and seasonal variation.

Bath Ankylosing Spondylitis Functional Index was not associated with vitamin D deficiency. The median BASFI score ${ }^{5.9}$ was used to stratify functional impairment into high and low BASFI subgroups. Significant association between deficiency and increasing BASDAI was present in those with high BASFI (ORadj 1.42; 95\% CI,

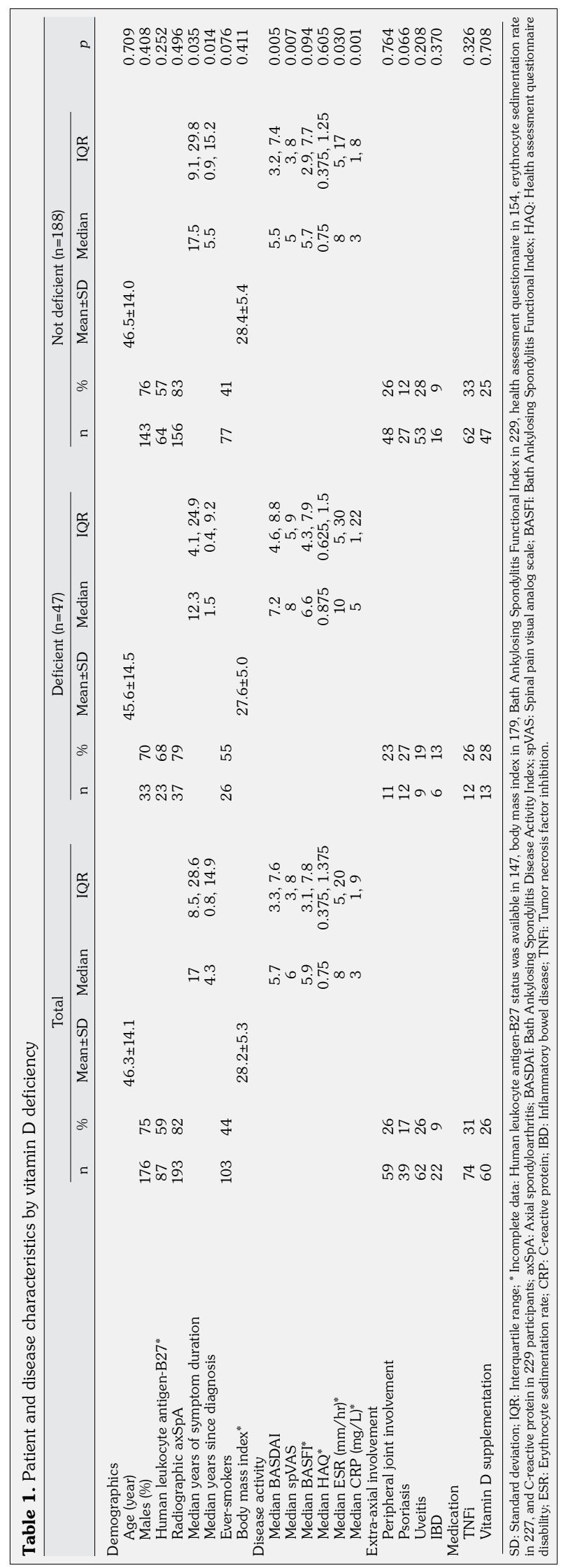


Table 2. Univariate and multivariable logistic regression models demonstrating associations between vitamin $\mathrm{D}$ deficiency and markers of disease severity

\begin{tabular}{|c|c|c|c|c|c|c|}
\hline & \multicolumn{2}{|c|}{ Univariate } & \multirow[b]{2}{*}{$p$} & \multicolumn{2}{|c|}{ Multivariable adjusted* } & \multirow[b]{2}{*}{$p$} \\
\hline & Odds ratio & $95 \% \mathrm{CI}$ & & Odds ratio & $95 \% \mathrm{CI}$ & \\
\hline BASDAI & 1.20 & $1.05,1.38$ & 0.006 & 1.23 & $1.06,1.41$ & 0.005 \\
\hline Spinal pain visual analogue scale & 1.17 & $1.04,1.31$ & 0.008 & 1.21 & $1.07,1.38$ & 0.003 \\
\hline BASFI** $^{* *}$ & 1.11 & $0.98,1.25$ & 0.096 & & & \\
\hline Erythrocyte sedimentation rate $(\mathrm{mm} / \mathrm{hr})^{* * *}$ & 1.01 & $1.00,1.03$ & 0.038 & 1.01 & $1.00,1.03$ & 0.077 \\
\hline C-reactive protein $(\mathrm{md} / \mathrm{L})^{* * *}$ & 1.03 & $1.01,1.04$ & 0.004 & 1.02 & $1.01,1.04$ & 0.005 \\
\hline
\end{tabular}

CI: Confidence interval; BASDAI: Bath Ankylosing Spondylitis Disease Activity Index; BASFI: Bath Ankylosing Spondylitis Functional Index; * Adjusted for age, sex, vitamin D supplementation, and seasonal variation; ${ }^{* *}$ Regression was performed with Bath Ankylosing Spondylitis Functional Index assessment in 229 erythrocyte sedimentation rate in 227 , and C-reactive protein in 229 participants.

1.04-1.94) but no significant association was seen in the subgroup with low BASFI (OR adj $0.92 ; 95 \%$ CI, 0.70-1.21).

Associations between vitamin $\mathrm{D}$ deficiency and increasing tertiles of BASDAI and spVAS are shown in Table 3. The highest tertile of BASDAI had a three-fold increased likelihood of vitamin D deficiency $\left(\mathrm{OR}_{\text {adj }} 3.1 ; 95 \% \mathrm{CI}\right.$, 1.27-7.55), compared to those in the lowest BASDAI subgroup. A greater than four-fold increase in likelihood of vitamin D deficiency was observed in patients of the highest spVAS tertile $\left(\mathrm{OR}_{\text {adj }} 4.3\right.$; 95\% CI, 1.61-11.4), compared to the spVAS reference group. Analysis for CRP was not statistically significant.

\section{DISCUSSION}

Vitamin D deficiency was observed in $20 \%$ of this cohort of axSpA patients, which is similar to that described in other axSpA populations. ${ }^{11}$ This crosssectional study has demonstrated a significant association between vitamin D deficiency and increased disease activity (BASDAI) in axSpA. Similar associations were also observed with level of spinal pain and inflammatory markers but no significant associations were seen with level of functional impairment. There was greater than three-fold increased likelihood of vitamin $\mathrm{D}$ deficiency observed in those with the highest tertiles of BASDAI and pain after adjustment for age, sex, vitamin D supplementation, and seasonal variation.

These results complement the findings from an earlier systematic literature review which identified that serum levels of $25(\mathrm{OH}) \mathrm{D}$ in axSpA patients were lower than healthy controls. ${ }^{3}$ Several studies included in this review reported inverse correlations between 25(OH)D levels and disease activity. Studies which failed to demonstrate such associations often employed suboptimal methodology. Firstly, there is substantial seasonal variation in serum $25(\mathrm{OH}) \mathrm{D}$ levels which should be accommodated for. Studies in the past have either neglected this or tried to overcome seasonal

Table 3. Association between vitamin D deficiency and increasing tertiles of disease activity and functional impairment (adjusted for age, sex, vitamin D supplementation, and seasonal variation)

\begin{tabular}{lcccc}
\hline & & & \multicolumn{2}{c}{ Vitamin D deficiency } \\
\cline { 3 - 5 } & Tertiles & $\mathrm{n}$ & OR $_{\text {adj }}$ 95\% CI \\
\hline Bath Ankylosing Spondylitis Disease Activity Index & $0-3.9$ & 80 & Reference group \\
& $4-7.2$ & 79 & 1.45 & $0.57,3.70$ \\
Spinal pain visual analog scale & $7.3-10$ & 76 & 3.10 & $1.27,7.55$ \\
& $0-4$ & 95 & Reference group \\
C-reactive protein & $5-8$ & 92 & 3.02 & $1.25,7.31$ \\
& $8.5-10$ & 48 & 4.29 & $1.61,11.4$ \\
& $0-1$ & 78 & Reference group \\
& $2-6$ & 75 & 1.13 & $0.46,2.77$ \\
ORadj: Adjusted odds ratio; CI: Confidence interval. & $7-132$ & 76 & 2.14 & $0.92,4.97$ \\
\hline
\end{tabular}


variation by sampling patients within a short time window. Secondly, many existing publications studied $25(\mathrm{OH}) \mathrm{D}$ as one of multiple variables in the context of bone metabolism in small axSpA cohorts, which can lead to both type 1 and 2 errors.

The strengths of the current study include use of sufficient sample size to study the association between vitamin D deficiency and BASDAI, with adjustment for important confounders. This included adjustment for seasonal variation using a sine function. In addition, as many of the variables, including $25(\mathrm{OH}) \mathrm{D}$, had non-Gaussian distributions, it was necessary to use statistical techniques that do not require normally distributed data or residuals. Deficiency was studied instead of absolute 25(OH)D levels as transformation of this strongly skewed variable made interpretation complex and difficult.

The sample size was not sufficient to draw firm conclusions in the function-stratified subgroups. However, the finding of a stronger association between BASDAI and vitamin D deficiency in the group of patients with high functional impairment is interesting. This may be more suggestive of vitamin $\mathrm{D}$ deficiency having an immunomodulating effect rather than simply being the result of high disease activity causing immobility and thus less UVB exposure and reduced 25(OH)D production. However, this is speculation and not supported by the results from this small sample. BASFI was not significantly associated with vitamin D deficiency despite its strong correlation with BASDAI/ spVAS.

Serum $25(\mathrm{OH}) \mathrm{D}$ is converted into its active metabolite $1,25(\mathrm{OH}) 2 \mathrm{D}$. This classically occurs in the kidney but can also be performed by $\mathrm{T}$ - and B-cells, macrophages and dendritic cells. ${ }^{2}$ $1,25(\mathrm{OH}) 2 \mathrm{D}$ is difficult to measure given its low serum concentration and short half-life. Serum $1,25(\mathrm{OH}) 2 \mathrm{D}$ may also have less relevance given that proposed immune mechanisms are likely auto-/paracrine. ${ }^{2}$ Nuclear vitamin D receptors are expressed by most immune cells. $1,25(\mathrm{OH}) 2 \mathrm{D}$ binds to vitamin $\mathrm{D}$ receptors which function as a ligand-activated transcription factor. In addition to well-known effects on calcium metabolism, these gene products have been reported to reduce proinflammatory pathways and promote those involved in immune tolerance. ${ }^{2}$ The pathology of axSpA is thought to involve elevated levels of IL23 and a subset of IL23-responsive entheseal T-cells. ${ }^{12} 1,25(\mathrm{OH}) 2 \mathrm{D}$ has been shown to inhibit both subunits of interleukin-23 (IL-23). ${ }^{13,14}$ IL-17 is another important cytokine in the IL-23-axis, and therapeutic inhibition of IL-17 has shown clinical efficacy. ${ }^{15}$ Treatment with vitamin $\mathrm{D}$ receptors agonists decreased IL-17 production in mice..$^{14,16}$

There are many factors associated with active axSpA that could result in a vitamin $\mathrm{D}$ deficient state. These include the presence of subclinical bowel inflammation, associated with active axSpA, that may impair absorption; although dietary contribution to vitamin D status is small. ${ }^{9}$ Vitamin $\mathrm{D}$ deficiency has been reported to correlate with chronic pain, ${ }^{17}$ which may contribute to symptoms of axSpA and increase patient-reported measures of disease activity.

Interestingly, vitamin D deficient patients had shorter median symptom duration and duration since diagnosis. This may be explained by the fact that patients with longer disease duration were significantly more likely to be commenced on vitamin D supplementation (data not shown). However, use of vitamin D supplementation did not appear to have an effect on 25(OH)D levels. This reinforces the need to distinguish supplementation from treatment doses, as the former is unlikely to be adequate in treating deficiency. ${ }^{4}$ Patients in this study only received supplementation at the time of assessment. Furthermore, vitamin D formulations vary in dose and compliance is well-known to be poor, especially when combined with calcium. ${ }^{18}$

Also interesting was the signal that deficient patients were more often smokers. This is in line with previous studies of smoking and vitamin D. ${ }^{19}$ Smoking and consequent systemic inflammation may affect vitamin D absorption and metabolism. Smoking status may also be a proxy for lower socioeconomic class and/or dietary quality.

Presence of psoriasis showed some association with vitamin D deficiency. This is consistent with other studies demonstrating greater prevalence of deficiency and lower serum levels of $25(\mathrm{OH}) \mathrm{D}$ in psoriasis and psoriatic arthritis compared with controls. ${ }^{20}$ Psoriasis is intimately linked with UVB and vitamin D both in treatment and possibly pathogenesis. ${ }^{20}$ Further detailed studies powered to explore these interesting results are required. 
This study has some limitations. Our axSpA cohort appears to have a low proportion of HLA-B27 positivity. HLA-B27 was not routinely tested at this center (note only in 63\%), and only ordered where clinical diagnosis was unclear (i.e. clinically obvious axSpA is likely to also be HLA-B27 positive but would not be tested). One other limitation of this study was that we did not use the more sensitive index Ankylosing Spondylitis Disease Activity Score. ${ }^{21}$ BASDAI is an established, reliable and valid disease activity measure in axSpA, but has been shown to be less sensitive to change in disease activity than Ankylosing Spondylitis Disease Activity Score in some studies. However, the fact that we were still able to detect significant associations between $25(\mathrm{OH}) \mathrm{D}$ and the Bath indices is a strength of the study. An association was also observed for CRP, giving some biological credence to the observation that disease activity is inversely associated with $25(\mathrm{OH}) \mathrm{D}$.

In conclusion, these results support the hypothesis that vitamin $\mathrm{D}$ deficiency has an immunomodulatory role in axSpA. However, the cross-sectional study design prevents detection of a causal relationship and it is not possible to know whether increased disease activity or vitamin $\mathrm{D}$ deficiency occurred first. Understanding whether vitamin $\mathrm{D}$ deficiency leads to increased disease activity in axSpA through immunomodulation is a complex problem that warrants further study. In addition, osteoporosis and vertebral fractures are important issues in axSpA, with the former accelerated by associated functional impairment. ${ }^{22}$ Patients with high BASDAI and spVAS have over three times the odds of being vitamin $\mathrm{D}$ deficient. It is therefore important that vitamin $\mathrm{D}$ deficiency is identified and treated in these patients to optimize bone health. The hypothesis that vitamin $\mathrm{D}$ deficiency may also cause more severe disease has clinical importance and is an area for future study, as targeting disease control by modulating vitamin $\mathrm{D}$ levels may be an attractive intervention to explore in axSpA.

\section{Acknowledgement}

We thank research nurses Helen Frankland and Ayren Mediana for recruiting patients. We also thank laboratory scientists Dr Andrew Cross and Jenny Hawkes for technical assistance.

\section{Declaration of conflicting interests}

The authors declared no conflicts of interest with respect to the authorship and/or publication of this article.

\section{Funding}

The authors received no financial support for the research and/or authorship of this article.

\section{REFERENCES}

1. Tam LS, Gu J, Yu D. Pathogenesis of ankylosing spondylitis. Nat Rev Rheumatol 2010;6:399-405.

2. Mora JR, Iwata M, von Andrian UH. Vitamin effects on the immune system: vitamins $\mathrm{A}$ and $\mathrm{D}$ take centre stage. Nat Rev Immunol 2008;8:685-98.

3. Zhao S, Duffield SJ, Moots RJ, Goodson NJ. Systematic review of association between vitamin D levels and susceptibility and disease activity of ankylosing spondylitis. Rheumatology (Oxford) 2014;53:1595-603.

4. Zhao S, Gardner K, Taylor W, Marks E, Goodson $\mathrm{N}$. Vitamin D assessment in primary care: changing patterns of testing. London J Prim Care (Abingdon) 2015;7:15-22.

5. Rudwaleit M, Jurik AG, Hermann KG, Landewé R, van der Heijde D, Baraliakos X, et al. Defining active sacroiliitis on magnetic resonance imaging (MRI) for classification of axial spondyloarthritis: a consensual approach by the ASAS/OMERACT MRI group. Ann Rheum Dis 2009;68:1520-7.

6. van der Linden S, Valkenburg HA, Cats A. Evaluation of diagnostic criteria for ankylosing spondylitis. A proposal for modification of the New York criteria. Arthritis Rheum 1984;27:361-8.

7. Maska L, Anderson J, Michaud K. Measures of functional status and quality of life in rheumatoid arthritis: Health Assessment Questionnaire Disability Index (HAQ), Modified Health Assessment Questionnaire (MHAQ), Multidimensional Health Assessment Questionnaire (MDHAQ), Health Assessment Questionnaire II (HAQ-II), Improved Health Assessment Questionnaire (Improved HAQ), and Rheumatoid Arthritis Quality of Life (RAQoL). Arthritis Care Res (Hoboken) 2011;63:4-13.

8. Sieper J, Rudwaleit M, Baraliakos X, Brandt J, Braun J, Burgos-Vargas $\mathrm{R}$, et al. The Assessment of SpondyloArthritis international Society (ASAS) handbook: a guide to assess spondyloarthritis. Ann Rheum Dis 2009;68:1-44.

9. Francis R, Aspray T, Fraser W, Gittoes N, Javaid K, Macdonald $\mathrm{H}$, et al. Vitamin D and Bone Health: A Practical Clinical Guideline for Patient Management: National Osteoposis Society; 2013. Available from: https://www.nos.org.uk/document.doc?id=1352.

10. Stolwijk AM, Straatman H, Zielhuis GA. Studying seasonality by using sine and cosine functions in 
regression analysis. J Epidemiol Community Health 1999;53:235-8.

11. Durmus B, Altay Z, Baysal O, Ersoy Y. Does vitamin $\mathrm{D}$ affect disease severity in patients with ankylosing spondylitis? Chin Med J (Engl) 2012;125:2511-5.

12. Sherlock JP, Joyce-Shaikh B, Turner SP, Chao CC, Sathe M, Grein J, et al. IL-23 induces spondyloarthropathy by acting on ROR- $\gamma \mathrm{t}+$ CD3+CD4-CD8- entheseal resident T cells. Nat Med 2012;18:1069-76.

13. Lemire JM, Archer DC, Beck L, Spiegelberg HL. Immunosuppressive actions of 1,25-dihydroxyvitamin D3: preferential inhibition of Th1 functions. J Nutr 1995;125:1704-8.

14. Daniel C, Sartory NA, Zahn N, Radeke HH, Stein $\mathrm{JM}$. Immune modulatory treatment of trinitrobenzene sulfonic acid colitis with calcitriol is associated with a change of a T helper (Th) 1/Th17 to a Th2 and regulatory $T$ cell profile. $J$ Pharmacol Exp Ther 2008;324:23-33.

15. Baeten D, Sieper J, Braun J, Baraliakos X, Dougados M, Emery P, et al. Secukinumab, an Interleukin-17A Inhibitor, in Ankylosing Spondylitis. N Engl J Med 2015;373:2534-48.

16. Penna G, Amuchastegui S, Cossetti C, Aquilano F, Mariani R, Sanvito F, et al. Treatment of experimental autoimmune prostatitis in nonobese diabetic mice by the vitamin D receptor agonist elocalcitol. J Immunol
2006;177:8504-11.

17. Knutsen KV, Brekke M, Gjelstad S, Lagerløv P. Vitamin $D$ status in patients with musculoskeletal pain, fatigue and headache: a cross-sectional descriptive study in a multi-ethnic general practice in Norway. Scand J Prim Health Care 2010;28:166-71.

18. Sunyecz JA. The use of calcium and vitamin $\mathrm{D}$ in the management of osteoporosis. Ther Clin Risk Manag 2008;4:827-36.

19. Brot C, Jorgensen NR, Sorensen OH. The influence of smoking on vitamin D status and calcium metabolism. Eur J Clin Nutr 1999;53:920-6.

20. Gisondi P, Rossini M, Di Cesare A, Idolazzi L, Farina S, Beltrami G, et al. Vitamin D status in patients with chronic plaque psoriasis. Br J Dermatol 2012;166:505-10.

21. Pedersen SJ, Sørensen IJ, Garnero P, Johansen JS, Madsen OR, Tvede N, et al. ASDAS, BASDAI and different treatment responses and their relation to biomarkers of inflammation, cartilage and bone turnover in patients with axial spondyloarthritis treated with TNF- $\alpha$ inhibitors. Ann Rheum Dis 2011;70:1375-81.

22. van der Weijden MA, van Denderen JC, Lems WF, Heymans MW, Dijkmans BA, van der HorstBruinsma IE. Low bone mineral density is related to male gender and decreased functional capacity in early spondylarthropathies. Clin Rheumatol 2011;30:497-503. 